\title{
Micro-channel Chemiluminescence Analysis Using a Peroxyoxalate Reaction that Works through Liquid-Liquid Interface Collapse under Laminar-Flow Conditions
}

\author{
Kazuhiko Tsukagoshi, ${ }^{* \dagger}$ Yoshiyuki Hattori,* Teruki HayashI,* Riichiro NaKaJIMa,* \\ Kenichi YAMASHITA,** and Hideaki MAEDA $* * * * * *$ \\ *Department of Chemical Engineering and Materials Science, Faculty of Science and Engineering, \\ Doshisha University, Kyotanabe, Kyoto 610-0321, Japan \\ **Micro- \& Nano-space Chemistry Group, Nanotechnology Research Institute, National Institute of Advanced \\ Industrial Science and Technology (AIST), 807-1 Shuku-machi, Tosu, Saga 841-0052, Japan \\ ***JST, CREST, 807-1 Shuku-machi, Tosu, Saga 841-0052, Japan
}

\begin{abstract}
An oxalate reagent-hydrogen peroxide-fluorescent compound chemiluminescence reaction, i.e., peroxyoxalate chemiluminescence, was introduced into micro-channel chemiluminescence analysis (MCCLA) to establish the concept of MCCLA through the direct observation of fluorescence and chemiluminescence using a fluorescence microscope-CCD camera and a microscope-CCD camera. It was confirmed from visual data that chemiluminescence in the MCCLA generated through the liquid-liquid interface collapsed during the course of molecular diffusion in the micro-channel. In addition, the visual data of chemiluminescence were transformed to line drawings on a computer to obtain chemiluminescence profiles. The chemiluminescence profiles were examined in detail at various flow rates and detection points; the relationship between the residence times and the chemiluminescence peak heights, or areas in the profiles, was easily represented as one smoothly changing reaction curve. Furthermore, the fluorescent compounds were detected with high sensitivity and good reproducibility in MCCLA by turning the syringe pumps on and off to produce determinable chemiluminescence signals; a photomultiplier tube was used as a detector. The chemiluminescence intensities in the signals of erythrosine, rhodamine B, Rose Bengal, fluorescein isothiocyanate, and eosin Y were examined; their intensities increased in this order, and eosin Y responded over the range of $1 \times 10^{-9}-1 \times 10^{-6} \mathrm{M}$ with a detection limit of $1 \times 10^{-9} \mathrm{M}$ $(S / N=3)$. Introducing of the peroxyoxalate chemiluminescence reaction into MCCLA can extend the analysis system to the analysis of various types of sample and applications incorporating fluorescence labeling techniques.
\end{abstract}

(Received May 30, 2008; Accepted August 15, 2008; Published November 10, 2008)

\section{Introduction}

Chemiluminescence (CL) has attracted a great deal of attention as an interesting and unique reaction and detection system in analytical chemistry. ${ }^{1-5} \mathrm{CL}$ analysis has a number of advantages: 1) high detection sensitivity, 2) wide linearity range of the signal response, 3) inexpensive reagents and apparatus, 4) easy and rapid measurement, and 5) no light sources or spectroscopes are required, which makes the instrument configuration very simple. CL has already been verified to be a high-performance detection method in capillary electrochromatography, capillary electrophoresis, microchip-capillary electrophoresis, ultramicro-flow systems, as well as various micro-total analysis systems ( $\mu$-TAS). ${ }^{6-10}$ Many types of reaction spaces have been proposed and investigated in CL analysis systems to achieve the above-mentioned CL advantages; however, they can be roughly classified into two types of reaction area, i.e., batch reaction and flow-mixing reaction types. ${ }^{11-15}$

We have been studying a specific CL reaction area around the

† To whom correspondence should be addressed.

E-mail: ktsukago@mail.doshisha.ac.jp liquid-liquid interface in a micro-channel under laminar-flow conditions. ${ }^{16-19}$ In this report, we call the analytical system taking advantage of the specific CL area micro-channel chemiluminescence analysis (MCCLA). MCCLA has a number of features as follows: 1) The CL in MCCLA is stably, continuously, and reproducibly observed with even very short residence times of less than $0.1 \mathrm{~s}$, where the residence time is decided by the distance from the junction of the reagents to the detection point as well as the reagent flow rates. ${ }^{17,18}$ That is, even CL characterized by a very fast reaction rate or very short lifetime can be measured with a definite CL intensity in MCCLA with the reagents fed into the micro-channel in a micro-reactor. 2) Controlling the reagent flows in a microchannel by turning the syringe pumps on and off (to start and stop reagent delivery) leads to specific CL signals, reflecting molecular diffusion around the liquid-liquid interface in a micro-channel without sample injectors. The CL signals obtained with such procedures make MCCLA, a useful quantitative analytical method. ${ }^{16-18}$ 3) Liquid-liquid interface collapse in a micro-channel can be achieved through only molecular diffusion without requiring mechanical stirring. It is difficult to examine chemical reactions around such a specific reaction area caused only by molecular diffusion. We can see 


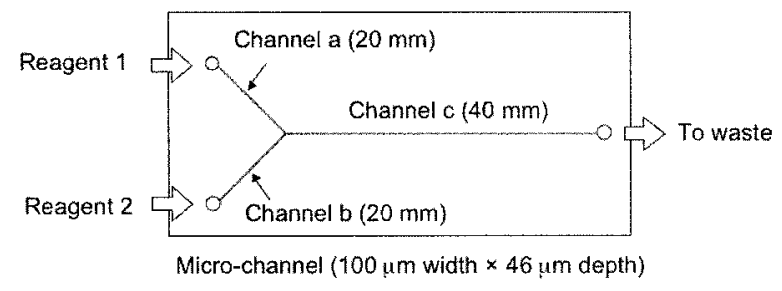

Micro-reactor 1 (size $30 \mathrm{~mm} \times 70 \mathrm{~mm}$; thickness $2 \mathrm{~mm}$ )

Fig. 1 Micro-reactor incorporating a micro-channel $(100 \mu \mathrm{m}$ in width $\times 46 \mu \mathrm{m}$ in depth); Micro-reactor 1 .

the chemical reaction behavior directly by investigating CL around such a specific reaction area in MCCLA. ${ }^{16-18}$ 4) Experiments to examine the effects of various conditions, such as temperature, magnetic fields, and applied voltage, on chemical reactions can be easily introduced into MCCLA, because the micro-channel is made in a $2-\mathrm{mm}$ thick microreactor. We examined the effects of magnetic fields on the CL in MCCLA by attaching two permanent magnets to the microchannel (micro-reactor). ${ }^{20}$ 5) Here, we will briefly discuss the future perspectives of MCCLA. Because MCCLA is performed around a liquid-liquid interface under laminar-flow conditions with molecule diffusion, the reaction area of MCCLA is the pseudo-in vivo chemical reaction type. We have reported on the effects of saliva on MCCLA using a singlet oxygen CL reaction. ${ }^{19}$

To date, we have studied MCCLA using the luminol-hydrogen peroxide-catalyst CL system $^{16}$ and a sodium hypochloritehydrogen peroxide singlet oxygen CL system ${ }^{17-19}$ through CL detection using a photomultiplier tube. In this study, the peroxyoxalate $\mathrm{CL}$ reaction was introduced into MCCLA; MCCLA was visually examined using a fluorescence microscope-CCD camera and a microscope-CCD camera to more firmly make up the concept of MCCLA. The peroxyoxalate $\mathrm{CL}$ reaction is one of the most useful CL reactions in analytical chemistry, ${ }^{21-23}$ since it can be used to detect various types of fluorescent compounds, including labeling reagents. The use of the peroxyoxalate CL reaction will expand the applicability of MCCLA for use with various analytes, including biomolecules, such as amino acids, peptides, protein, saccharides, and nucleic acids.

\section{Experimental}

\section{Chemicals}

Water was purified with an Elix UV 3 (Millipore, Tokyo, Japan). All of the reagents used were commercially available and of special grade, and were purchased from the following sources: bis[2-(3,6,9-trioxadecanyloxycarbonyl)-4-nitrophenyl]oxalate (TDPO), eosin Y, rhodamine, erythrosine, and Rose Bengal were from Wako Pure Chemical Industries (Osaka, Japan); acetonitrile and hydrogen peroxide were from Nacalai Tesque (Kyoto, Japan); and fluorescein isothiocyanate (FITC) was from Dojindo Laboratories (Kumamoto, Japan).

\section{Micro-reactors and analytical procedure}

Figures 1 and 2 show illustrations of the micro-reactors used in the present study, incorporating a micro-channel $(100 \mu \mathrm{m}$ in width $\times 46 \mu \mathrm{m}$ in depth) made of quartz glass (Fig. 1, Microreactor 1), which was purchased from Institute of Microchemical

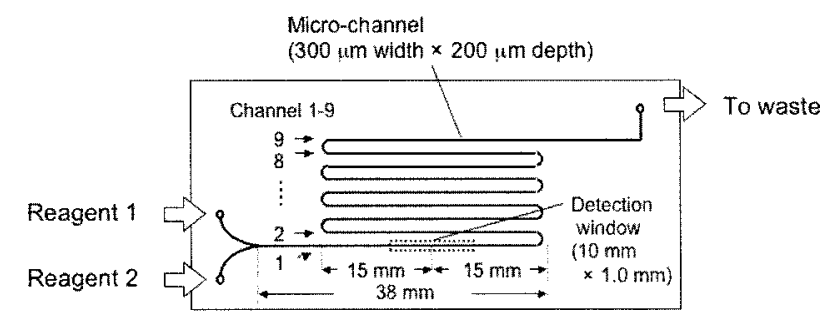

Micro-reactor 2 (size $30 \mathrm{~mm} \times 70 \mathrm{~mm}$; thickness $2 \mathrm{~mm}$ )

Fig. 2 Micro-reactor incorporating a micro-channel $(300 \mu \mathrm{m}$ in width $\times 200 \mu \mathrm{m}$ in depth); Micro-reactor 2 .

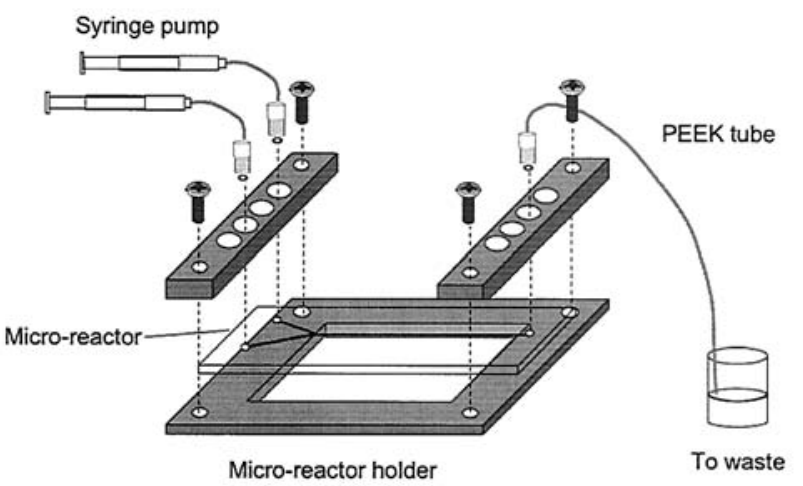

Fig. 3 Illustration of the setup of the micro-reactor in the microreactor holder.

Technology (Type; ICC-SY05) and a micro-channel (300 $\mu \mathrm{m}$ in width $\times 200 \mu \mathrm{m}$ in depth) made of quartz glass and silicon (Fig. 2, Micro-reactor 2). A micro-channel in Micro-reactor 2 was fabricated by a dry-etching process on a silicon plate. The fabrication was carried out by an ICE Plasma System (EIS-700, Elionix Inc.; Tokyo, Japan) using $\mathrm{C}_{4} \mathrm{~F}_{8}$ and $\mathrm{SF}_{6}$ gases. An anodic bonding system was employed for bonding between a quartz glass and a channel-fabricated silicon plate.

Micro-reactor 1 was used for obtaining visual data, and photographs by a CCD camera, while Micro-reactor 2 was used for obtaining CL signals by a photomultiplier tube, because of its long channel length, including channel curves, and was not used for visual data because of its opaque nature (though only from one side) due to the silicon material. Micro-reactors were set up in a micro-reactor holder, as shown in Fig. 3. Reagent 1 was $2 \mathrm{mM}$ TDPO and $200 \mathrm{mM}$ hydrogen peroxide in an acetonitrile solution. Reagent 2 was a fluorescent compound solution in $10 \mathrm{mM}$ phosphate buffer ( $\mathrm{pH} 9.0)$. The two reagent solutions were delivered into the micro-channels by a microsyringe pump (MF-9090; Bioanalytical Systems Inc., West Lafayette, IN). They were joined at the junction point in a micro-reactor, and subsequently fed to the micro-channel.

The fluorescence and $\mathrm{CL}$ in Channel $\mathrm{c}$ in Micro-reactor 1 were observed visually with a fluorescence microscope (BX51; Olympus, Tokyo, Japan) using an $\mathrm{Hg}$ lamp and filter (UMWIB2, ex. 460 - $495 \mathrm{~nm}$, em. >510 nm)-CCD camera (JKTU53H, Toshiba, Tokyo, Japan) and microscope (BX51; Olympus)-CCD camera. In addition, the obtained visual CL data were transformed to line drawings to express the color depth on a computer. Furthermore, the resulting CL in Channels 1 - 9 in the Micro-reactor 2 was detected with a photomultiplier tube (H5783; Hamamatsu Photonics Co. Ltd., Shizuoka, Japan) 


\section{a) Fluorescence}

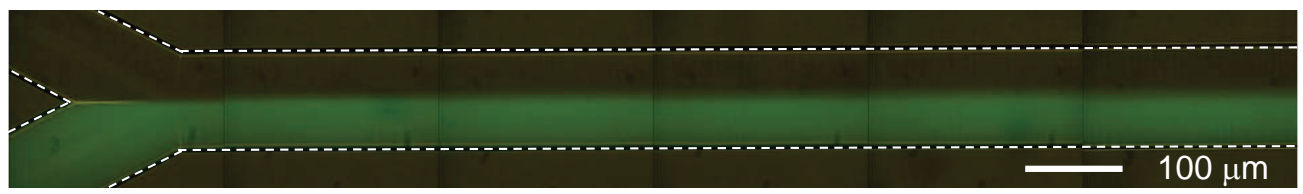

\section{b) $C L$}

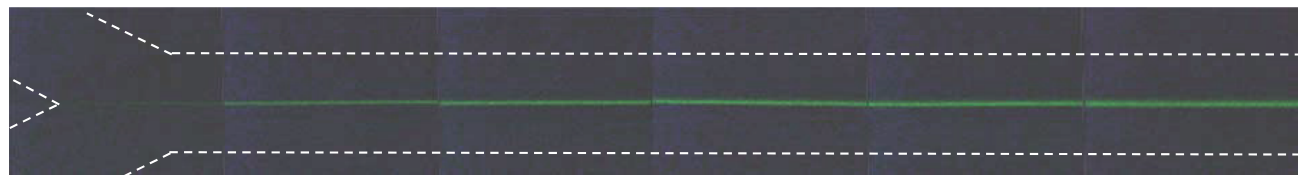

Flow direction $\longrightarrow$

Fig. 4 Fluorescence and CL in Channel c in Micro-reactor 1. Conditions: a) Fluorescence observed through the fluorescence microscope-CCD camera: Reagent 1, $10 \mathrm{mM}$ phosphate buffer ( $\mathrm{pH} 9.0$ ); Reagent 2, $10 \mathrm{mM}$ phosphate buffer ( $\mathrm{pH}$ 9.0) including $1 \times 10^{-5} \mathrm{M}$ eosin $\mathrm{Y}$; each flow rate, $50 \mu 1 \mathrm{~min}^{-1}$. b) CL observed through the microscope-CCD camera: Reagent 1, acetonitrile including $2 \mathrm{mM}$ TDPO and $200 \mathrm{mM}$ hydrogen peroxide; Reagent 2, $10 \mathrm{mM}$ phosphate buffer $(\mathrm{pH} 9.0)$ including $1 \times 10^{-2} \mathrm{M}$

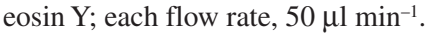

located under the micro-reactor, measured with a CL detector (Model EN-21; Kimoto Electric Inc., Osaka, Japan), and treated with an integrator (Chromatopac C-R6A; Shimadzu Co., Kyoto, Japan). As micro-flows in the micro-channels in fluorescence and CL were observed through a microscope-CCD camera to reach a stationary state quickly, we started the measurements without any waiting time.

\section{Results and Discussion}

Observation of fluorescence and $C L$ in the micro-channel through a fluorescence microscope-CCD camera and microscope-CCD camera

The peroxyoxalate $\mathrm{CL}$ reaction is one of the most important $\mathrm{CL}$ reactions used in various flow systems. ${ }^{24-26}$ Oxalate reagents, such as bis(2,4,6-trichlorophenyl)oxalate (TCPO) and TDPO, react with hydrogen peroxide to give a dioxetane compound as an intermediate, which interacts with fluorescent compounds. Energy transfer occurs from the dioxetane compound to the fluorescent compound through complex formation between them, and the excited fluorescent compound drops to the ground state, emitting CL. Oxalate reagents are usually dissolved in organic solvents, such as acetonitrile, and are easily decomposed when dissolved in aqueous solution. In this study, the peroxyoxalate CL reaction was introduced into MCCLA.

Figure 4 shows visual data of fluorescence and CL in Channel $\mathrm{c}$ in Micro-reactor 1 through the fluorescence microscope-CCD camera and the microscope-CCD camera. Figure 4a shows observations of the fluorescence obtained under the following conditions: Reagent 1, $10 \mathrm{mM}$ phosphate buffer ( $\mathrm{pH} 9.0$ ); Reagent 2, $10 \mathrm{mM}$ phosphate buffer $(\mathrm{pH} 9.0)$ containing $1.0 \times$ $10^{-5} \mathrm{M}$ eosin $\mathrm{Y}$. Figure $4 \mathrm{~b}$ shows the observations of $\mathrm{CL}$ obtained under the following conditions: Reagent 1, acetonitrile solution containing $2 \mathrm{mM}$ TDPO and $200 \mathrm{mM}$ hydrogen peroxide; Reagent 2, $10 \mathrm{mM}$ phosphate buffer ( $\mathrm{pH} 9.0$ ) containing $1.0 \times 10^{-2} \mathrm{M}$ eosin $\mathrm{Y}$.

The results shown in these figures clearly confirmed that in MCCLA the two reagent solutions flowed in the micro-channel, forming a liquid-liquid interface under laminar-flow conditions (the Reynolds numbers were roughly calculated to be less than 10 ), and then the CL reaction occurred around the interface through molecular diffusion to yield CL.

\section{CL profiles in the micro-channel at various detection points and flow rates}

We examined CL profiles at various detection points $(5-\mathrm{mm}$ intervals along Channel $\mathrm{c}$ in Micro-reactor 1$)$ and flow rates (2, $5,10,20,50$, and $100 \mu \mathrm{min}^{-1}$; they were the flow rates for the one micro-syringe pump). The CL profiles were obtained from CL photographs obtained with the microscope-CCD camera, changing the visual data to line drawings on a computer in the following way. The obtained visual CL data, i.e., the photograph shown in Fig. $4 \mathrm{~b}$, was transformed to line drawings to express the color depth on a computer. The observed colors on the photographs were divided into RGB (red, green, and blue); naturally, the photograph in Fig. 4b was mainly occupied with green, since eosin Y emits CL light at a wavelength of $c a .560$ $\mathrm{nm}$. The green color depths were expressed numerically as digital data on a computer. Finally, the numbers were standardized to the line-drawing data to give CL profiles. As examples, the obtained CL profiles at flow rates of $50 \mu 1 \mathrm{~min}^{-1}$ and at various detections points are shown in Fig. 5; the distances from the junction point to the each detection area are shown at the upper region of each CL profile in Fig. 5; also, the residence times indicating the times needed from the junction point to the each detection area are indicated at the lower part of the each CL profile in Fig. 5.

As can be seen in Fig. 5, the CL peaks were observed in the CL profiles around the liquid-liquid interface in the microchannel. The CL peak heights and the CL peak areas are plotted against the residence times at various detection points and flow rates in Figs. $6 a$ and $6 b$, respectively. Although they were observed at the same detection point with good reproducibility (relative standard deviation of the CL peak height and the area were $<2 \%$ and $<5 \% ; n=7-9$, respectively), it seemed difficult to set the micro-channel at the same detection point for microscope observation repeatedly, maybe leading to a few discrepancies in the plots in Figs. $6 \mathrm{a}$ and $6 \mathrm{~b}$. However, from the 
$50 \mu 1$ min $^{-1}$

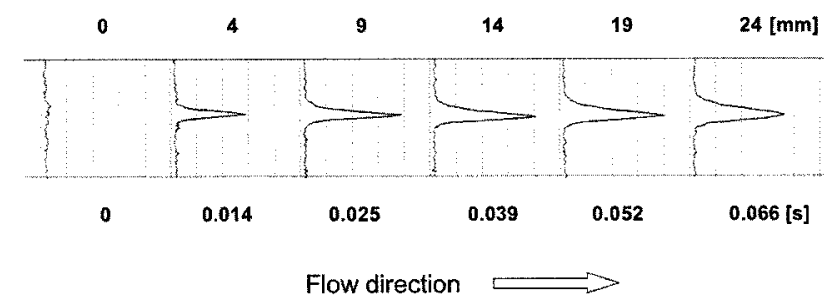

Fig. 5 CL profiles obtained for visual data of the microscope-CCD camera at various detection points in Channel $\mathrm{c}$ in Micro-reactor 1 Each flow rate of $50 \mu 1 \mathrm{~min}^{-1}$.

figures, the relationship between the residence times and the CL peak heights or areas in the profiles were easily represented as one smoothly changing reaction curve; this meant that the observed CL depended on the residence times, not on the flow rates. The relationships shown in Fig. 6 were consistent with that the flow rates (or the axial linear velocities) in a microchannel under laminar-flow conditions did not naturally influence the liquid-liquid interface or its collapse, and that, consequently, liquid-liquid collapse in a micro-channel was achieved through only molecular diffusion, as described in Introduction.

The CL peak heights or peak areas increased with the residence times ( $\mathrm{ca} .0 .005 \mathrm{~s}-$ ), and gave maximum values around $c a .0 .08 \mathrm{~s}$ for the CL peak heights or $c a .0 .3 \mathrm{~s}$ for the peak areas as shown in Fig. 6. Also, they subsequently decreased with the residence time (- $2 \mathrm{~s})$; consumption of the reactive chemical species in the micro-channel must lead to the disappearance of CL. Since molecular diffusion first occurred with the residence time around the interface in MCCLA, leading to the chemical reaction and the emission of CL, the phenomenon, as shown in Fig. 6, that the maximum peak height appeared earlier than the maximum peak area might usually be observed in MCCLA, if the reaction rates are not extremely low. We are now interested in such CL observations in MCCLA, and will study them in detail in the next stage.

\section{CL signals of a TDPO-hydrogen peroxide-fluorescent compound} with a photomultiplier tube in MCCLA

Several groups have attempted to apply the CL reaction in micro-channels fabricated in micro-reactors for quantitative analyses..$^{27-29}$ For example, $\mathrm{Cr}(\mathrm{III})^{27}$ and epinephrine ${ }^{28}$ were detected with CL reactions of luminol and lucigenin, respectively. It should be emphasized that most CL detection systems in micro-reactors require an external sample-injection device, which results in the formation of a sample plug in the micro-channel. The sample plug leads to peak formation in the CL profile as output. The observation of a peak signal is very useful for quantitatively detecting analytes that participate in the $\mathrm{CL}$ reaction. However, a sample injection device to make a sample plug sometimes requires additional parts, such as a sample loop, injector, carrier line, and a delivery system, necessitating laborious procedures to handle them. Furthermore, any mechanism to mix a sample plug with a CL reagent may require additional devices to be fabricated in the micro-reactor.

We have proposed a method for determining CL signals due to a CL reaction in a micro-channel fabricated within a microreactor without sample plug formation, which is one of the merits of MCCLA, as mentioned in Introduction. Some interesting CL signals were observed with high sensitivities by a)

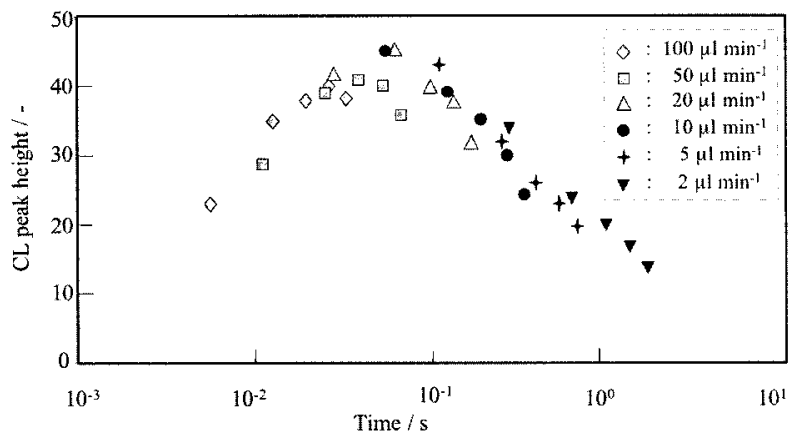

b)

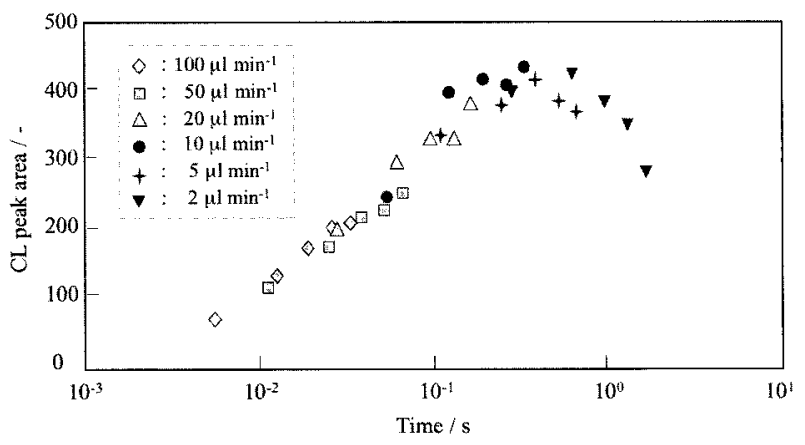

Fig. 6 Relationships between a) the residence times and the CL peak heights as well as b) the residence times and the CL peak areas in the CL profiles in Channel $\mathrm{c}$ in Micro-reactor 1. Conditions: Reagent 1, acetonitrile including $2 \mathrm{mM}$ TDPO and $200 \mathrm{mM}$ hydrogen peroxide; Reagent 2, $10 \mathrm{mM}$ phosphate buffer $(\mathrm{pH} 9.0)$ including $1 \times 10^{-2} \mathrm{M}$ eosin $\mathrm{Y}$; detection point, 5-mm intervals along the microchannel; each flow rate, $2,5,10,20,50$, or $100 \mu \mathrm{min}^{-1}$.

photomultiplier tube detection through collapse of the liquidliquid interface based on laminar-flow in a micro-channel. ${ }^{16-19}$

A typical CL signal in the present MCCLA method using the peroxyoxalate CL reaction is shown in Fig. 7, which was obtained by turning the syringe pumps on and off using Microreactor 2, which had a long channel including channel curves (the detection window of Channel 1 shown in Fig. 2 was used). As can be seen in the figure, the CL responded rapidly to turning the syringe pumps on and off; the CL appeared and disappeared with operation of the syringe pumps. In MCCLA, laminar-flow that begins with turning on the syringe pumps results in a liquidliquid interface, yielding intense $\mathrm{CL}$ around it. When the reagents are fed into the micro-channel, $\mathrm{CL}$ is observed stably and continuously under the same conditions of the mixing and reaction time, and the CL disappeared rapidly after the pumps were turned off.

Furthermore, we examined the effects of the curve in the micro-channel on the CL intensity using Micro-reactor 2. The experiments were carried out at various detection windows in Channels $1-9$, as shown in Fig. 2 at flow rates of 10, 20, 50, and $100 \mu \mathrm{min}^{-1}$. The CL signals were observed in detection windows of Channels $1-4$, and then not observed in those of Channels 5 - 9. Figure 8 shows the relationship between the residence times and the $\mathrm{CL}$ intensities. The residence time when using Micro-reactor 2 was estimated by using the microchannel length from the junction point to the middle of the detection window. Each plot was average for 7-9 measurements (relative standard deviation, $<2 \%$ ). The $\mathrm{CL}$ intensities decreased with increasing residence times; all plots showed the same 


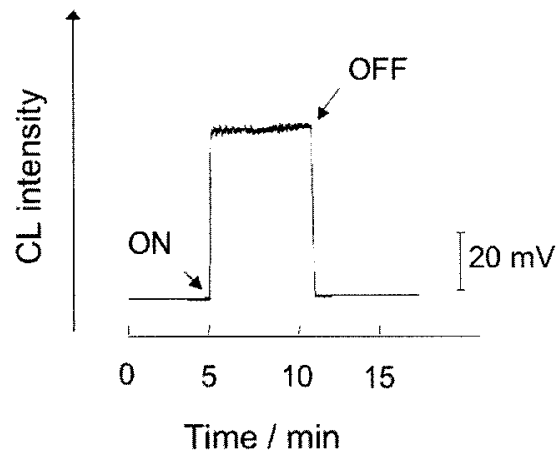

Fig. 7 CL signals of fluorescent compounds in MCCLA using the peroxyoxalate reaction. "On" and "off" indicate turning the syringe pumps on and off, respectively. Conditions: Reagent 1 , acetonitrile including $2 \mathrm{mM}$ TDPO and $200 \mathrm{mM}$ hydrogen peroxide; Reagent 2 , $10 \mathrm{mM}$ phosphate buffer $(\mathrm{pH} 9.0)$ including $1 \times 10^{-7} \mathrm{M}$ eosin $\mathrm{Y}$ detection window, Channel 1 in Micro-reactor 2; each flow rate, $50 \mu 1$ $\min ^{-1}$.

decreasing curve, indicating that there was no effect of the curve in the micro-channel on the $\mathrm{CL}$ intensities in the present MCCLA method.

\section{Detection of fluorescent compounds with MCCLA}

As described above, the CL intensities decreased with increasing residence times under the present conditions. However, the noise level of the CL intensities increased with decreases in the residence time (the data not shown). Judging from the noise level and the CL intensity, subsequent experiments were carried out with a flow rate of $50 \mu \mathrm{l} \mathrm{min}{ }^{-1}$, corresponding to the one micro-syringe pump.

Various fluorescent compounds, erythrosine, rhodamine B, Rose Bengal, FITC, and eosin Y, were examined at a concentration of $1 \times 10^{-7} \mathrm{M}$. These compounds showed relative CL intensities of 15, 24, 38, 47, and 100, respectively, with that of eosin $\mathrm{Y}$ given an arbitrary value of 100 . In addition, eosin $\mathrm{Y}$ was determined over the concentration range of $1 \times 10^{-9}-1 \times$ $10^{-6} \mathrm{M}$ with a detection limit of $1 \times 10^{-9} \mathrm{M}(S / N=3$; relative standard deviation, $<2 \% ; n=7-9)$. The quantitative analysis was shown to have high sensitivity and reproducibility.

\section{Conclusions}

We have been studying the CL reaction around the liquid-liquid interface in micro-channels under laminar-flow conditions since 2004. Here, we call this micro-channel CL analysis (MCCLA). MCCLA is performed in such a specific reaction space, which is not assigned to the usual reaction spaces, including batch and flow-mixing reaction spaces. The features of MCCLA described in the Introduction section had been demonstrated by investigations of luminol and singlet oxygen $\mathrm{CL}$ with a photomultiplier tube. In this study, peroxyoxalate CL using fluorescent compounds was introduced into MCCLA. The introduction made the concept of MCCLA clear through the observation of fluorescence and CL with a microscope and a CCD camera. In addition, various fluorescent compounds including FITC as a labeling reagent could be determined with high sensitivity and reproducibility with a photomultiplier tube by turning the syringe pumps on and off. MCCLA has begun to attract interest from the viewpoint of analytical chemistry, while taking advantage of the novel reaction area.

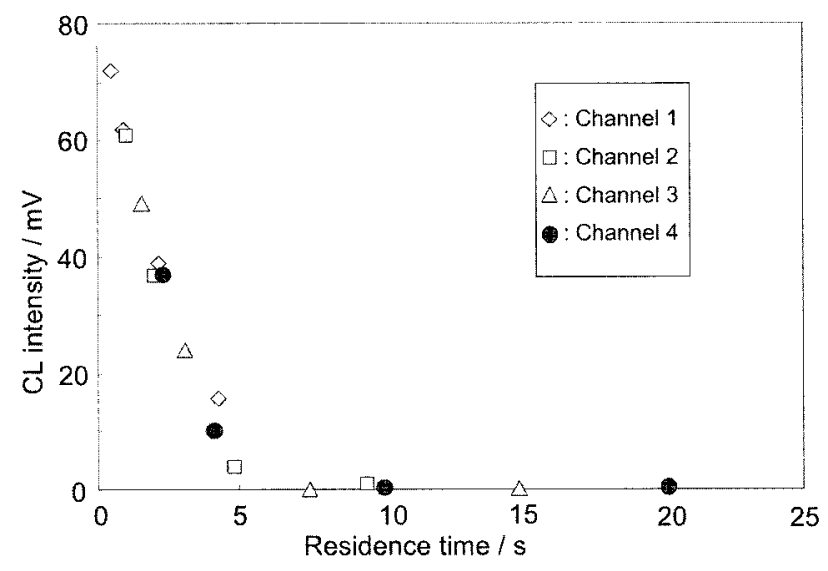

Fig. 8 Relationship between the residence times and the CL intensities at various flow rates and detection points. Conditions: Reagent 1, acetonitrile including $2 \mathrm{mM}$ TDPO and $200 \mathrm{mM}$ hydrogen peroxide; Reagent 2, $10 \mathrm{mM}$ phosphate buffer $(\mathrm{pH} 9.0)$ including $1 \times$ $10^{-7} \mathrm{M}$ eosin $\mathrm{Y}$; detection point, Channels $1-4$ in Micro-reactor 2; each flow rate, $10,20,50$, or $100 \mu 1 \mathrm{~min}^{-1}$.

\section{Acknowledgements}

This work was supported by a Grant-in-Aid for Scientific Research (C) from the Ministry of Education, Culture, Sports, Science, and Technology, Japan. This study was also supported by the Academic Frontier Research Project on New Frontier of Biomedical Engineering Research of the Ministry of Education, Culture, Sports, Science, and Technology, Japan.

\section{References}

1. K. Tsukagoshi, K. Nakahama, and R. Nakajima, Anal. Chem., 2004, 76, 4410.

2. M. Miro, J. M. Estela, and V. Cerda, Anal. Chim. Acta, 2005, 541, 57.

3. K. A. Fletcher, S. O. Fakayode, M. Lowry, S. A. Tucker, S. L. Neal, I. W. Kimaru, M. E. McCarroll, G. Patonay, P. B. Oldham, O. Rusin, R. M. Strongin, and I. M. Warner, Anal. Chem., 2006, 78, 4047.

4. C. A. Marquette and L. J. Blum, Anal. Bioanal. Chem., 2006, 385, 546.

5. L. G.-Gracia, A. M. G.-Campana, J. J. S.-Chinchilla, J. F. H.-Perez, and A. G.-Casado, TrAC, 2005, 24, 927.

6. X.-B. Yin and E. Wang, Anal. Chim. Acta, 2005, 533, 113.

7. X. Huang and J. Ren, $\operatorname{Tr} A C, \mathbf{2 0 0 6}, 25,155$.

8. K. Tsukagoshi, N. Jinno, and R. Nakajima, Anal. Chem., 2005, 77, 1684.

9. S. Goetz and U. Karst, Anal. Bioanal. Chem., 2007, 387, 183.

10. S. Motomizu and Z.-H. Li, Talanta, 2005, 66, 332.

11. B. Haghighi and R. Dadashvand, Anal. Lett., 2007, 40, 2425.

12. K. Tsukagoshi, T. Nakamura, and R. Nakajima, Anal. Chem., 2002, 74, 4109.

13. K. Tsukagoshi, M. Tahira, and R. Nakajima, J. Chromatogr., A, 2005, 1094, 192.

14. B. Horstkotte, O. Elsholz, and V. Cerda, J. Flow Inject. Anal., 2005, 22, 99.

15. K. Tsukagoshi, K. Sawanoi, and R. Nakajima, Talanta, 
2006, 68, 1071.

16. K. Tsukagoshi, K. Ikegami, R. Nakajima, K. Yamashita, and H. Maeda, Chem. Lett., 2004, 33, 1178.

17. K. Tsukagoshi, K. Fukumoto, K. Noda, R. Nakajima, K. Yamashita, and H. Maeda, Anal. Chim. Acta, 2006, 570, 202.

18. K. Tsukagoshi, K. Fukumoto, R. Nakajima, K. Yamashita, and H. Maeda, Talanta, 2007, 72, 607.

19. K. Tsukagoshi, K. Fukumoto, R. Nakajima, K. Yamashita, and H. Maeda, Sci. Eng. Rev. Doshisha Univ., 2007, 48, 134.

20. R. Utsumi, R. Nakajima, K. Tsukagoshi, K. Yamashita, and H. Maeda, 88th Spring Meeting of the Chemical Society of Japan, 26 - 30 March, 2008, Rikkyo University, Tokyo, Japan.

21. M. Tsunoda and K. Imai, Anal. Chim. Acta, 2005, 541, 13.
22. K. Tsukagoshi, K. Tsuge, and R. Nakajima, Anal. Sci., 2007, 23, 739.

23. H. Shen, Q. Fang, and Z.-L. Fang, Lab Chip, 2006, 6, 1387.

24. Y. Sun, M. Wada, O. Al-Dirbashi, N. Kuroda, H. Nakazawa, and K. Nakashima, J. Chromatogr., B, 2000, 749, 49.

25. K. Tsukagoshi, Y. Shikata, R. Nakajima, M. Murata, and M. Maeda, Anal. Sci., 2002, 18, 1195.

26. K. Tsukagoshi, T. Kameda, M. Yamamoto, and R. Nakajima, J. Chromatogr., A, 2002, 978, 213.

27. Y. Xu, F. G. Bessoth, J. C. T. Eijkel, and A. Manz, Analyst, 2000, 125, 677.

28. T. Kamidate, T. Kaide, H. Tani, E. Makino, and T. Shibata, Anal. Sci., 2001, 17, 951.

29. Z. Zhang, D. He, W. Liu, and Y. Lv, Luminescence, 2005, $20,377$. 